\title{
Reflectance of Biological Turbid Tissues under Wide Area Illumination: Single Backward Scattering Approach
}

\author{
Guennadi Saiko and Alexandre Douplik \\ Department of Physics, Ryerson University, Toronto, ON, Canada M5B 2K3 \\ Correspondence should be addressed to Alexandre Douplik; douplik@ryerson.ca
}

Received 19 December 2013; Accepted 3 February 2014; Published 10 March 2014

Academic Editor: Victor Loschenov

Copyright ( 2014 G. Saiko and A. Douplik. This is an open access article distributed under the Creative Commons Attribution License, which permits unrestricted use, distribution, and reproduction in any medium, provided the original work is properly cited.

\begin{abstract}
Various scenarios of light propagation paths in turbid media (single backward scattering, multiple backward scattering, banana shape) are discussed and their contributions to reflectance spectra are estimated. It has been found that a single backward or multiple forward scattering quasi-1D paths can be the major contributors to reflected spectra in wide area illumination scenario. Such a single backward scattering (SBS) approximation allows developing of an analytical approach which can take into account refractive index mismatched boundary conditions and multilayer geometry and can be used for real-time spectral processing. The SBS approach can be potentially applied for the distances between the transport and reduced scattering domains. Its validation versus the KubelkaMunk model, path integrals, and diffusion approximation of the radiation transport theory is discussed.
\end{abstract}

\section{Introduction}

Propagation of light in biological turbid tissues is a complex interplay between scattering and absorption. Light propagation in biological tissues is important for calculation of the exact amount of light in tissue, that is, the light dose for estimating thermal damage of biological tissues. The propagation of light in biotissue is also important for diagnostics technologies. The diffuse approximation and Monte Carlo simulations are widely used for these purposes $[1,2]$.

If we know optical properties of the media, then the light distribution within the media or the tissue reflectance or transmittance can be calculated by various methods [1]. With the advancements of computational power, Monte Carlo (MC) simulations [2] become the most common approach. It is also the most versatile and accurate approach, which can give an answer with required accuracy. The most common task in optical diagnostics however is to solve an inverse problem and reconstruct optical properties of the tissue from a certain reflectance (and less typically transmittance) spectrum. To solve it, various methods have been developed including inverse MC [1], inverse addingdoubling [3], and spatially[4] and spectrally resolved [5] diffuse reflectance methods. Some of them are applicable to very particular measurements (e.g., inverse adding-doubling method for single and double integrating sphere geometry); others (lookup MC tables, diffuse approximation) can be applied to certain simplified geometries (finite slab or semiinfinite slab). In real life, however, most of surface tissues are characterized by multilayered geometries and internal reflection due to the refraction index mismatched boundary conditions, which significantly complicate solving the inverse problem by existing techniques. Thus, a simplified analytical approach, which can facilitate processing of experimental data of light scattering processes into biotissues in realistic geometries, is of great importance.

The related question is to understand clear physical picture of light propagation within the media. In particular, we are interested in estimation of the sampling depth and the closely related question which we can actually see in the reflectance spectra (photons which entered the biotissue nearby (short pass) or diffuse photons, which entered far from the detector (long pass)). The most clear physical picture can be obtained using the path integrals approach to photon migration [6]. This analytical method is based on finding a classical path [7] for a nondissipating system and then calculating the path integral for all possible trajectories using Feynman formalism. The method can be used to find 
approximate solutions for forward and inverse problems in semi-infinite slab geometries. However, due to computational difficulties it is problematic to apply this approach in realistic geometries with refraction index mismatched boundary conditions, multilayered structures, and so forth.

Reflection spectra depend on particular illumination scenario. There are four major illumination scenarios: wide beam diffuse, wide beam collimated, point collimated (e.g., laser beam) and point diffuse (e.g., light through optical fiber) illumination. In medical biooptics, wide beam collimated and point diffuse (e.g., fiber source) illuminations are the most common illumination modalities used in therapeutic applications. The wide and point collimated beam illuminations are used or shortly will be used in quickly emerging diagnostic modality of photoacoustics [8]. In daily life we typically see the reflectance from the tissues under wide beam diffuse illumination (e.g., overcast conditions).

Thus, the question can be transformed into the following one: which volume exactly can we interrogate at different illumination scenarios?

Obviously, the diffusion is an important mechanism of light propagation in turbid media [1]. However, the light propagation in the vicinity of the light source (within the transport length) does not follow the diffuse approximation [9]. Moreover, some skin marks, such as birthmarks, scars or other skin inhomogeneity are seen with sharp borders, which indicates that the optical paths contribute in reflectance not only diffusively.

Existing models (diffuse approximation, Kubelka-Munk, and Monte Carlo simulations) do not address this question directly. The interrogation depth can be approximated from the light distribution; it is still unclear what is the typical optical path for photons measured by a detector. Monte Carlo code can be amended [10] to address this question, although it is difficult to process numerical simulations for multilayered geometries close to real time. Thus, a simple analytical model, which can address this question, will be very useful for interpretation of results.

By the way, this question is important not only for biological tissue optics but also for other turbid media applications. In particular, in paper and paint industry applications their typical geometry (dull colour layer parallel to an opaque plane substrate) is pretty similar to the typical geometry in tissue optics (e.g., papillary dermis over subcutaneous fat). Moreover, similar to surface tissues, paper is also a complex structure consisting of cellulose fibers, fillers, and additives (including chromophores).

The purpose of this paper is to develop a simplified analytical approach elucidating the physical picture of scattering processes in turbid tissues and providing interpretation of experimental results, particularly in tissues with high absorption and within a small distance (comparable to the transport length, $\left.I_{t}=1 /\left(\mu_{a}+\mu_{s}^{\prime}\right)\right)$ from the source. We show how this method can be used for estimating reflectance of tissue and sampling depth.

The paper is composed as follows: first, we develop an analytical approach to the light propagation in the turbid tissues; then, we compare predictions with diffuse approximation, Kubelka-Munk theory and path integrals approaches; finally, we discuss the physical picture of scattering in the tissue based on comparison of these models.

\section{Theory}

2.1. Individual Scattering Event. Individual scattering events in body tissues are primarily attributed to intracellular organelles (mitochondria, nuclei, etc.) with the size comparable to the photon wavelength (Mie regime [1]). These scattering events are highly anisotropic and primarily directed forward. We will be interested in probability of backward (with scattering angle $\theta>\pi / 2$ ) scattering $p$. This probability can be calculated directly from the Mie theory; however, for illustration purposes as one of the probability density functions characterizing the angular distribution of scattering we will use the Heiney-Greenstein scattering phase function [11], which is routinely used for light propagation in biotissues:

$$
p(g, \theta)=\frac{1}{2} \frac{1-g^{2}}{\left(1+g^{2}-2 g \cos \theta\right)^{3 / 2}} .
$$

Here $g$ is "the mean cosine" of scattering, $\langle\cos \theta\rangle$. If $g=0$, we have isotropic scattering; $g=1$ or -1 corresponds to fully forward or fully backward scattering, respectively. The light scattering in tissues is strongly anisotropic with $g$ ranging from 0.785 (dermis) to 0.995 (blood).

From (1) we can estimate the probability of backward scattering $p$-the probability that photon will be scattered with $\theta>\pi / 2: p(g)=\int_{\pi / 2}^{\pi} p(g, \theta) \sin \theta d \theta$ :

$$
p(g)=\frac{1}{2} \frac{1-g^{2}}{g}\left[\frac{1}{\sqrt{1+g^{2}}}-\frac{1}{1+g}\right] .
$$

On Figure 1 the probability of backward scattering $p(g)$ is plotted as function of "average cosine" $g$ together with the probability of backward scattering in $1 \mathrm{D}$ model $(g=$ $\langle\cos \theta\rangle=\cos 0 *(1-p)+\cos \pi * p=1-2 p$-dotted line $)$.

It can be seen that the probability of backward scattering for body tissues $p$ is not bigger than 0.05 (dermis) and typical value of $p$ in tissues with blood is even smaller.

2.2. Multiple Scattering in Turbid Tissues. Let us consider the path of a photon that started and finished on the surface of tissue (reflection spectrum). We can classify the optical paths based on the number of backscattering events (as defined in the previous section) along this path. Optical paths obtained by Monte Carlo simulations can be visually grouped into 3 major classes: (1) quasi-1D paths with one or more backward scatterings, (2) a "sausage" or "banana shape" paths with multiple tilted forward scatterings, and (3) pure diffuse paths with multiple forward and backward scattering events. Backward scattering and "banana shape" paths are depicted in Figure 2. We can distinguish between paths with one backward scattering and multiple (more than 1) backward scatterings, but we also can consider paths with tilted forward scattering as a subset of the diffuse paths. Thus, the amended classification can be presented as follows. 


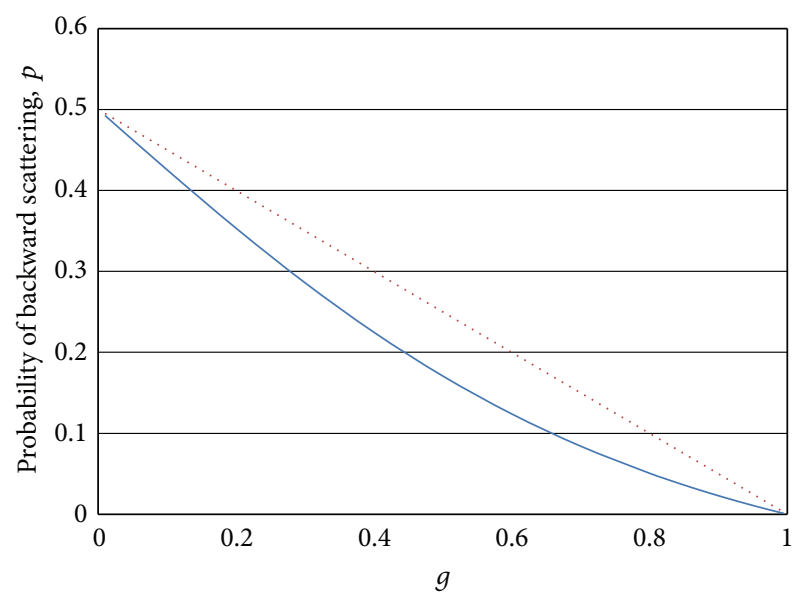

Figure 1: Probability of backward scattering $p(g)$ in the HeineyGreenstein model (solid line) and 1D model (dotted line) as a function of $g$.

(1) Path with one backward scattering (see Figure 2(a)).

(2) Path with multiple backward scatterings (see Figure 3).

(3) Diffuse path, including path with tilted forward scatterings (see Figure 2(b)).

Let us consider contributions of each of these scenarios in the total reflected flux. To calculate this contribution we consider homogeneous medium with coefficients of absorption $\mu_{a}$ and scattering $\mu_{s}$, respectively. To simplify our calculations we also consider normal (or almost normal) incidence of the external light flux.

2.2.1. Path with One Backward Scattering. Let us consider that each scattering event occurs on the distance $l_{s}=1 / \mu_{s}$ (this assumption can be relaxed; however, it will complicate the calculations). Such as forward and almost forward scattering events dominate substantially, so we can consider the following quasi-1D model (the path is almost ballistic; however, it can slightly deviate in transverse directions): if the photon was scattered $n$ times forwardly (with probability $1-p$ ) and then was scattered backwardly (with probability $p$ ), then to reach the surface again it must undergo additionally $n$ forward scattering events (see Figure 2(a)). The probability of this $n-1-n$ path is $p(1-p)^{2 n}$. However, here we did not take into account yet that during this path the photon flux undergoes also absorption.

To take into account absorption let us consider the photon flux propagation between two scattering events. The intensity of out coming flux $I_{\text {out }}=I_{\text {in }} \exp \left(-\mu_{a} l_{s}\right)=I_{\text {in }} \exp \left(-\mu_{a} / \mu_{s}\right)$. So, we can attribute an absorption multiplier $a=\exp \left(-\mu_{a} / \mu_{s}\right)$ to each pathway between two closest scattering events.

Thus in previously considered case of $n-1-n$ scatterings we have to multiply scattering probability $p(1-p)^{2 n}$ on probability to survive in absorption between $2 n+1$ scattering events: $a^{2 n+2}$. So, the total contribution of this path to the total backward flux will be $p(1-p)^{2 n} a^{2 n+2}$.
It is easy to calculate the total contribution of all paths with single backward scattering (coefficient of reflectance based on superposition of the paths with single backward scattering events):

$$
\begin{aligned}
R_{1} & =p a^{2}+p(1-p)^{2} a^{4} \cdots+p(1-p)^{2 n} a^{2 n+2} \cdots \\
& =\frac{p a^{2}}{1-(1-p)^{2} a^{2}} .
\end{aligned}
$$

We can also develop continuous version of our approach. For this reason we in (3) switch from summation to integration: $\sum_{n=0}^{\infty} \Rightarrow \int d n$. Thus, if we consider a semifinite tissue, then we can integrate from 0 to infinity, and our integral is equal to

$$
R_{1}=\frac{p a^{2}}{2\left(\left(\mu_{a} / \mu_{s}\right)-\ln (1-p)\right)}
$$

2.2.2. Path with Multiple Backward Scattering. It is easy to see that if we exclude purely diffuse paths and limit ourselves to quasi-1D quasiballistic model, then the total number of backward scattering events must be odd. So, a diagram with three backward scattering events will be the next order one (see Figure 3).

These lowest order diagrams will be proportional to $p^{3} a^{6}$. To each diagram with the total path length $2 N$ we can assign a weight $W(2 N, 3)$ the number of different diagrams with the same length $2 N$ and 3 backward scatterings. This weight can be estimated from the geometry of this path. For our $n_{1}-1-n_{2}-1-n_{3}-1-n_{4}$ scattering path (here $n_{i}$ is the $i$-th path length between two backward scattering events) we can write $n_{1}+n_{3}=n_{2}+n_{4}=N$. Thus, we have $(N-2)^{2}$ nonequivalent diagrams (such as $1<n_{1}, n_{4}<N$ ). And the contribution of these diagrams can be approximated as $(N-2)^{2} p^{3}(1-p)^{2 N-4} a^{2 N}$. It is easy to see that for strongly anisotropic body tissues with blood we can neglect them in the future. A more accurate estimation of multiscattering contribution is provided in the Appendix. However, given that $p \ll 1$, we can expect that for $\mu_{a} / \mu_{s}>0.01$, the impact of all $p^{2 l+1}$, where $l \geq 1$ is rather small. For small $g$ (less than 0.5 which does not represent significant interest for biological tissues), multiple backward scattering can have measurable impact; however, the validity of quasi-1D model for $g<0.5$ is very questionable and this impact will likely be masked by the contribution of diffuse paths. We will discuss applicability of this quasi-1D model later.

2.2.3. Diffuse Path. Contribution of diffuse paths is well known in diffusion theory and can be calculated using various approaches. We can make some estimations of reflectance near the light entry point by visualizing light propagation as a random walk on cubic lattice with the unit size $l_{s}^{\prime}=1 / \mu_{s}^{\prime}[12]$. Thus, if the light entered the tissue at point $(x, y, 0)$, then it travels to $(x, y, 1)$ and from that point it occurs random walk through the media. However, to exit tissue in the close vicinity of the entry point it should travel again through $(x, y, 1)$ to $(x, y, 0)$. To take into account absorption and scattering for 


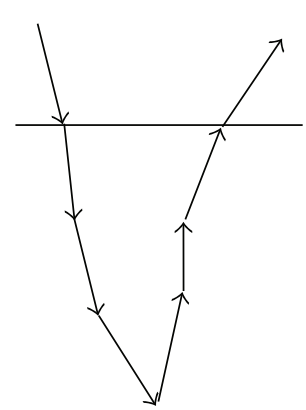

(a)

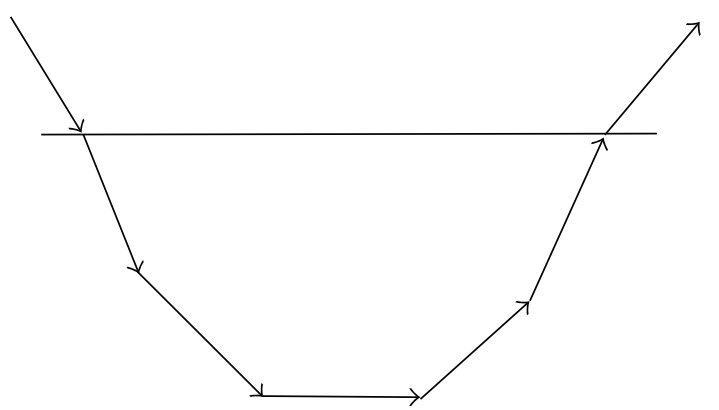

(b)

FIGURE 2: Paths with backward scattering (a) and "tilted" forward scatterings (b).

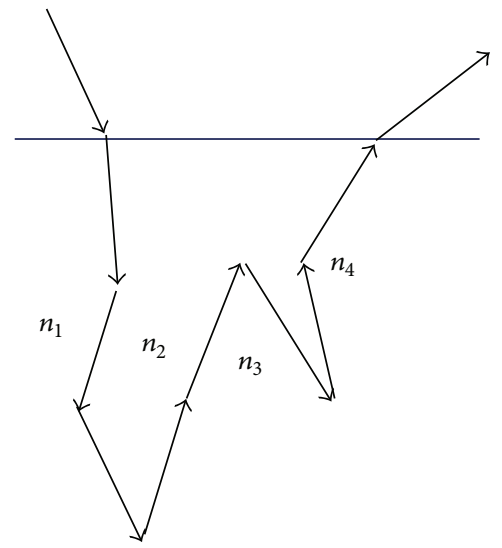

FIGURE 3: 3-fold backward scattering.

each edge of the lattice we can assign attenuation multiplier $\exp \left(-\left(\mu_{a}+\mu_{s}^{\prime}\right) l_{s}^{\prime}\right)=\exp \left(-1 / a^{\prime}\right)$, where $a^{\prime}=\mu_{s}^{\prime} /\left(\mu_{s}^{\prime}+\mu_{a}\right)$ is the reduced albedo. So, the reflectance at the light entry point $(x, y, 0)$ in the lattice model will be $R_{d}=\exp \left(-2 / a^{\prime}\right)(1 / 6+C)$, where $1 / 6$ is attributed to the shortest path $((x, y, 0) \rightarrow$ $(x, y, 1) \rightarrow(x, y, 0))$ and $C$ is the contribution of all paths with nonzero length which started and finished at $(x, y, 1)$. It is easy to see that to reach the surface the photon has to undergo $2 /(1-g)$ scattering events. Thus, in the case of small reduced albedo (e.g., $a^{\prime}<0.67$ if the absorption is high or $g$ is close to 1 in case of moderate absorption) the impact of diffuse paths can be quite low.

Thus, we have found that the single backward scattering can dominate among scattering mechanisms in turbid tissues under certain conditions, such as high absorption. A more accurate comparison of areas of applicability of diffuse approximation and SBS will be presented later, when we estimate the penetration depth.

\section{Results}

Now, we can apply the developed single backward scattering model (SBS) to calculate tissue reflectance and sampling depth.
3.1. Diffuse Reflectance. We will calculate tissue reflectance in SBS model and compare it with the K-M, diffuse approximation, and path integrals models. To compare models we will consider a semi-infinite media with matched boundary conditions.

3.1.1. Kubelka-Munk Model. One of the standard approaches to calculate reflectance in the geometry with homogeneous and totally diffuse illumination is the Kubelka-Munk model (K-M model) [13]. The K-M model has been widely used for multiple-scattering calculations in paper, paint, plastic, and textile industries for many years mostly due to its simple use and its acceptable prediction accuracy [14]. The K-M model works best for optically thick materials where more than $50 \%$ of light is reflected and less than $20 \%$ is transmitted.

Using the standard notation: $s$-scattering per unit length, $k$-absorption per unit length the reflection coefficient $R$ for the semi-infinite media is equal to

$$
R_{\mathrm{KM}}=1+\frac{k}{s}-\sqrt{\left(1+\frac{k}{s}\right)^{2}-1}
$$

It is easy to see that in terms of our model $k$ is the coefficient of absorption $\mu_{a}$, while $s$ is the coefficient of backward scattering per unit length. Thus, $s$ is equal to the scattering coefficient $\mu_{s}$ times the probability of backward scattering $p: s=p \mu_{s}$

3.1.2. Path Integrals. It was found by Perelman et al. [6] that the steady-state diffuse reflectance for semi-infinite media using path integrals formalism is given by a formula

$$
R_{\mathrm{PI}}=\exp \left[-2\left(\beta_{\mathrm{eff}} \frac{-\ln a}{1-g}\right)^{1 / 2}\right]
$$

where $a$ is the albedo $\left(a=\mu_{s} /\left(\mu_{s}+\mu_{a}\right)\right)$ and $\beta_{\text {eff }}$ is the numeric coefficient $\left(\beta_{\text {eff }}=2.17\right.$ and 2.47 for $g=0.757$ and 0.908, resp.). 
3.1.3. Diffuse Approximation. For collimated light beam illumination developed by Reynolds et al. [15] and Groenhuis et al. [16] in diffusion dipole model [17] the reflectance is given by

$$
R_{d}=\frac{\mu_{s}^{\prime}}{2 \mu_{t}^{\prime}} \exp \left(-\mu_{\mathrm{eff}}\left(z_{0}+2 h\right)\right),
$$

where $z_{0} \approx 1 / \mu_{t}^{\prime}$ and $h \approx 2 / 3 \mu_{t}^{\prime}$ for refractive index matching (here $\mu_{t}^{\prime}=\mu_{a}+\mu_{s}^{\prime}$ ).

The tissue reflectance predicted by this model is significantly smaller (see Figure 4) than those of provided by K$\mathrm{M}$, path integrals, and SBS, which means that this approach can be likely used just for spatially resolved geometries. The simplified expression 8.7 from [9] (index matching at the surface)

$$
R_{d}=\frac{a^{\prime}}{1+2\left(1-a^{\prime}\right)+(5 / 3) \sqrt{3\left(1-a^{\prime}\right)}}
$$

where $a^{\prime}=\mu_{s}^{\prime} /\left(\mu_{s}^{\prime}+\mu_{s}\right)$ is the reduced albedo, provides much more reasonable approximation.

On Figure 4 one can see the total diffuse reflectance as a function of $\mu_{a} / \mu_{s}$ ratio for different models: diffuse approximation model (8) (squares), SBS (rhomboids), path integrals (triangles), Kubelka-Munk (crosses), and diffusion dipole model (stars).

3.2. Sampling Depth. By definition the expression for the reflectance of tissue in single backward scattering approximation (3) is the sum of infinite number of terms. We can compare this expression with the contribution into reflectance of the first $m$-terms (scattering layers):

$$
R_{1, m}=\frac{p a^{2}}{1-(1-p)^{2} a^{2}}\left(1-(1-p)^{2 m} a^{2 m}\right) .
$$

So, if we limit ourselves by some threshold criteria (say we are interested in $1-\alpha=95 \%$ of total contribution) we can easily calculate the number of the most contributing $m$-term:

$$
(1-p)^{2 m} a^{2 m}=\alpha
$$

Thus,

$$
\begin{aligned}
m & =\frac{\ln (\alpha)}{2 \ln (a(1-p))} \\
& =\frac{\ln (\alpha)}{2\left(-\mu_{a} / \mu_{s}+\ln (1-p)\right)} \approx \frac{-\ln (\alpha)}{2\left(\mu_{a} / \mu_{s}+p\right)} .
\end{aligned}
$$

On Figure 5 we can see the dependence of light penetration depth as a function of $\mu_{a} / \mu_{s}$ for different $\alpha$ and $g$.

So, it is easy to see that information is being gathered from relatively thin surface scattering layer which is not deeper than $0.5-1 \mathrm{~mm}$, while the major part of information propagates from the top 2-3 transport depths $(0.1 \mathrm{~mm})$. Therefore, $2-5$ upper scattering layers $(m$-terms) contribute more than $50 \%$ in the total reflected flux. Also, the higher absorption is (or the lower $g$ ), the higher contributors the upper layers are.

Based on the previous discussion we can estimate the area of applicability of the single backward scattering approach. In order to do it, we take into account that for the quasi-1D model the transversal component of scattering expected to be much smaller than the scattering along the initial path. If we take into account that $g$-factor can be used as a proxy of the transverse component, then the longitudinal component after $m$ scatterings will be $g^{m}$ and thus applicability of the theory can be formulated as $1-g^{m}$.

\section{Discussion}

Let us discuss how these results can be applied to various illumination geometries. Comparison between different models gives us important insights about scattering scenarios in the media. For instance, the difference between Kubelka-Munk (single + multiple backward scatterings) and single backward scattering (SBS) approaches gives an estimation of impact of multiple backward scatterings.

4.1. Point Illumination with a Collimated Light. This geometry is directly addressed by all 4 models. This configuration is a typical measurement setup for numerous optical spectroscopy studies in clinics [1]. From Figure 5 it can be seen that the Kubelka-Munk model approximates well the diffuse reflectance calculated using path integrals and diffuse model in a wide range of parameters. SBS gives results close to Kubelka-Munk and path integrals within $\mu_{a} / \mu_{s}=0.01-0.1$ range, particularly for high anisotropy. It means that the single back scattering dominates in this range, while within the ultralow absorption range $\left(\mu_{a} / \mu_{s}=0.001-0.01\right)$ the other mechanisms dominate, namely, multiple backward scattering and diffusion.

As the diffuse approximation model predicts lower reflectance, one can expect that in the close vicinity of the light source (within the transport length, $l<1 /\left(\mu_{a}+\mu_{s}^{\prime}\right)$ ), the backward scattering scenarios will dominate and the primary part of the reflectance spectra will be contributed by the backward scattering. For the sites located further away from the light source, the diffuse approximation scenario will dominate.

4.2. Broad Illumination with a Collimated Light. This geometry (with a beam diameter wider than the transport length, $d>1 /\left(\mu_{a}+\mu_{s}^{\prime}\right)$ and collection from the illuminated area) can be considered as a superposition of multiple collimated point sources; thus, most results from the previous discussion are directly transferable. In particular, we can expect that the backward scattering will contribute more than the diffuse paths approaching from the remote part of the photon paths. Thus, in this scenario in case of high absorption $\left(a^{\prime}<0.67\right)$ primarily we collect backward scattered photons.

As it has been just shown the sampling depth is rather small, and the major impact is attributed to only a couple of surface layers ( $m$-terms). 


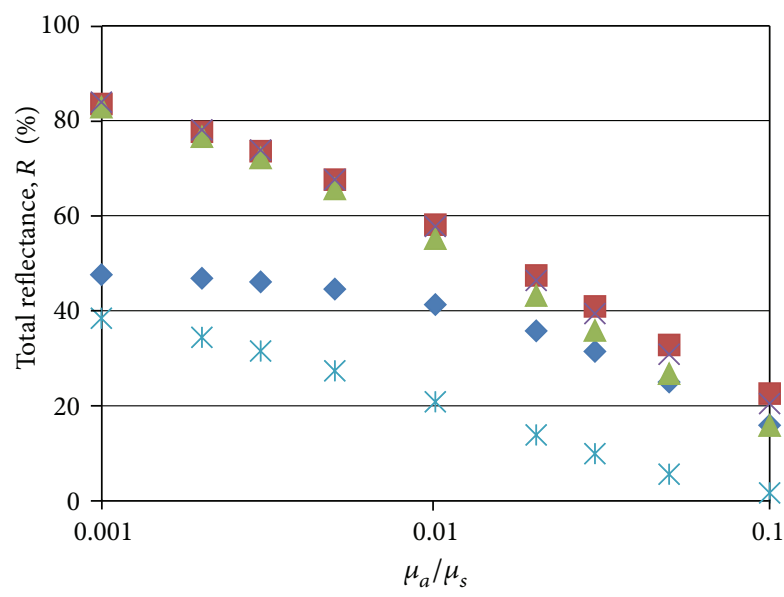

(a)

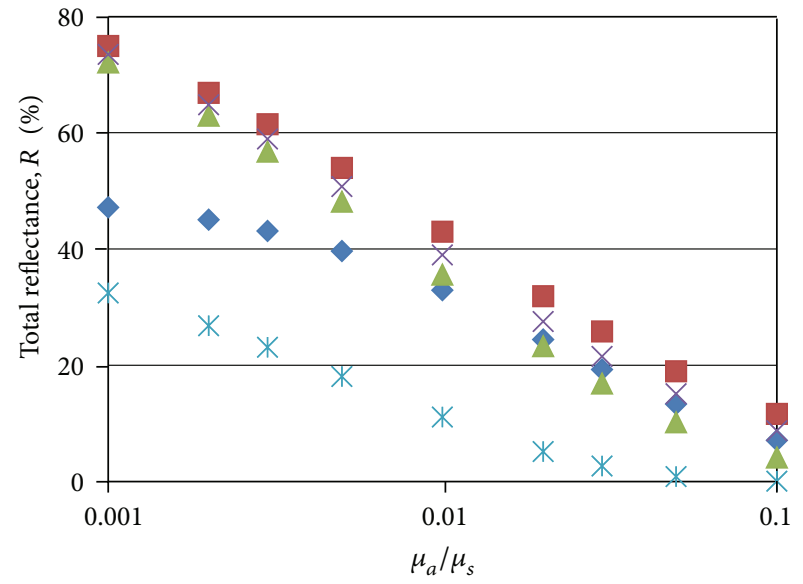

(b)

Figure 4: Total diffuse reflectance as a function of $\mu_{a} / \mu_{s}$ ratio for different models $(g=0.757$ (a), $g=0.908$ (b)): diffuse approximation (8) (squares), single back scattering model (SBS) (rhomboids), path integrals (triangles), Kubelka-Munk (crosses), and diffusion dipole model (7) (stars).

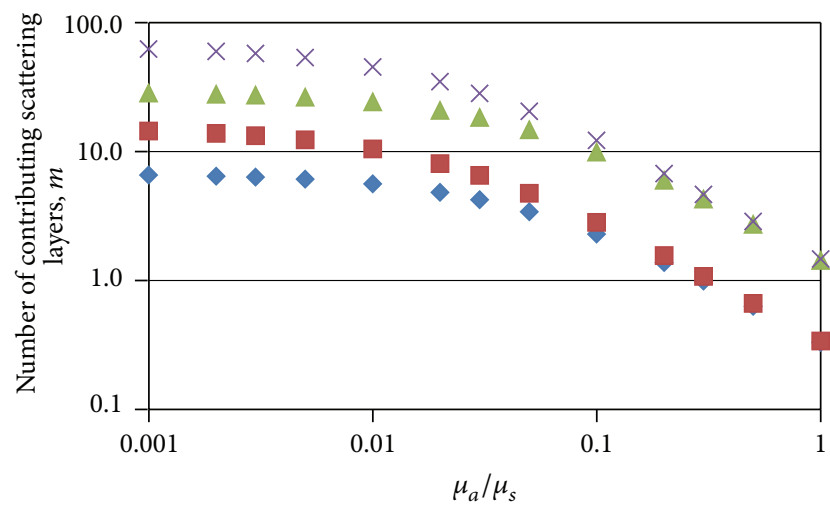

FIGURE 5: Number of contributing $m$-terms (scattering layers or transport depths) as a function of $\mu_{a} / \mu_{s}$ ratio for different $\alpha$ and $g$. $\alpha=0.5, g=0.8$ (rhomboids), $\alpha=0.5, g=0.9$ (squares), $\alpha=0.05$, $g=0.8$ (triangles), $\alpha=0.05$, and $g=0.9$ (crosses).

Thus, based on this discussion we can expect that SBS can be applicable for interpretation of spectra obtained by endoscopic measurements with wide area illumination. In this case, blood, which is highly absorbing in visual spectra, is much closer to the surface than in skin, and backward scattering paths may dominate over longer diffuse paths.

4.3. Single Backward Scattering Approach versus KubelkaMunk Model. These models are relatively close to each other (e.g., they both consider scattering as primarily forward and backward, they both can consider layered geometries, and they both can take into account realistic phase function or probabilities can be calculated directly from the Mie theory). The major difference consists in that the Kubelka-Munk model does not take into account the refractive index mismatched boundary conditions, although Saunderson took the internal reflection on the border with the opaque substrate into account [18]. However, the mismatched boundary conditions can be naturally integrated into SBS approach through additional reflection coefficients on the media layer interfaces.

The advantage of Kubelka-Munk model is that it incorporates multiple backward scattering. Consequently, in some specific geometries and conditions this model can be used to gauge the applicability of the single backward scattering (SBS) model.

It is easy to see (Figure 4) that the single backward scattering model matches Kubelka-Munk predictions perfectly for $\mu_{a} / \mu_{s}=0.02-0.3$. Thus, we can conclude that we can neglect the multiple backward scattering events for this range of parameters and the single backward scattering approach is applicable for biological tissues with $\mu_{a} / \mu_{s}>0.02$.

\subsection{The Single Backward Scattering Model and Diffuse Ap-} proximation. SBS and the scattering-dominant limit of Radiation Transport Model are complimentary to each other, such as they have different areas of applicability (see Figure 6).

The diffusion scattering approach is not applicable on the distances shorter than inversed reduced scattering coefficient $1 / \mu_{s}^{\prime}$. It is related to the fact that light propagation on the short distances (due to highly anisotropic nature of scattering) cannot be considered as truly random walk processes, and consequently the law of large numbers is not applicable here. The diffusion approximation is more applicable for the photon paths with more than $1 /(1-g)$ scattering events, after which the photon does not "remember" its original direction and scattering can be considered isotropic. However, this number in case of tissues with high blood content can be quite big (compare 200 for pure blood with 5 for dermis). For shorter distances, the photon propagation more resembles ballistic transfer than random walk process. The presented single backward scattering model can incorporate also features typical for short distances, and consequently is applicable for such shorter distances as well. 


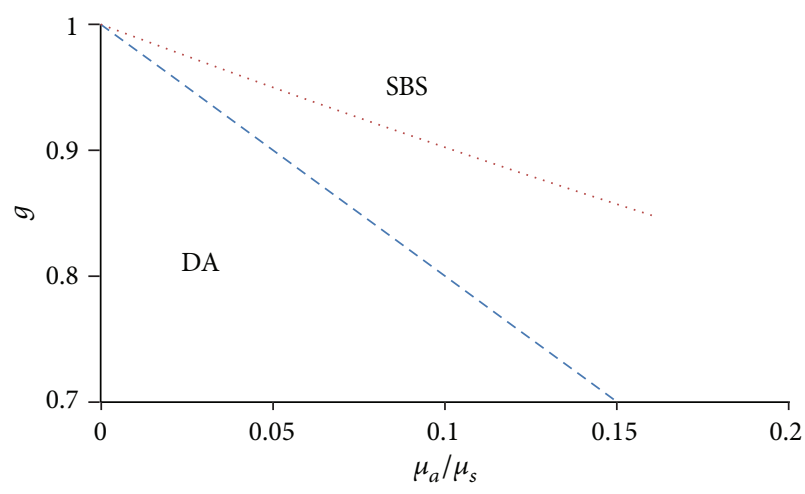

FIgURE 6: Areas of applicability of diffuse approximation (DA) and single backward scattering (SBS) models for biological tissues. Dashed line is $a^{\prime}=0.67$ and dotted line is $g^{m}=0.7$ (see (11) for definitions).

Also, it is known that diffuse approximation gives good agreement with Monte Carlo when $g<0.99$ and reduced albedo $a^{\prime}>0.67$ With higher absorption, the diffuse approximation predicts much higher reflectance than MC. Longer optical paths, typical for diffusion, have smaller impact than the backscattering paths. This is the area where SBS is particularly applicable to fill out the shortage in analytical approach tool spectrum.

\section{Conclusions and Future Work}

Single backward scattering approximation allows developing an analytical approach which can take into account mismatched boundary conditions and multilayer geometry and can be used for real-time spectral processing.

The single backward scattering model is probably applicable on distances shorter than $1 / \mu_{s}^{\prime}$ from the source in point illumination by collimated light and at certain scenarios in broad illumination by collimated light. The SBS approach can be potentially applied for the distances between the transport and reduced scattering domains-the remaining "grey" area where an analytical approach is in great demand.

Monte Carlo and experimental verification will be in focus of our future work.

\section{Appendix}

\section{Exact Calculations for 3-, and 5-Fold Backward Scattering}

Now we consider case $l=1$ (3-fold backward scattering; see Figure 3). For this geometry we have $n_{1}+n_{3}=N, n_{2}+n_{4}=N$, where $n_{1}, n_{2}, \ldots, n_{4}>0$. For each $n_{1}>1, n_{2}$ can vary from 1 to $n_{1}-1$. Thus, we have $W(2 N, 3)=\sum_{n=1}^{N-1}(n-1)$ nonequivalent paths, which is equal to $(1 / 2)(N-1)(N-2)$.

If we define new variables $x=a(1-p), k=N-2$, we have an exact expression for the impact of 3-fold backward scattering:

$$
R_{3}=p^{3} a^{4} \sum_{k=0}^{\infty} \frac{1}{2} k(k+1) x^{2 k}
$$

This expression can be rewritten as

$$
R_{3}=\frac{p^{3} a^{3} x}{4} \sum_{k=1}^{\infty} 2 k(k+1) x^{2 k-1} .
$$

It is easy to see that this expression can be transformed into

$$
R_{3}=\frac{p^{3} a^{3} x}{4} \frac{d}{d x} \sum_{k=1}^{\infty}(k+1) x^{2 k} .
$$

Taking into account that the sum in above expression is the expansion of $\left(1 /\left(1-x^{2}\right)^{2}\right)-1$, we have a transformed expression for (A.1):

$$
R_{3}=\frac{p^{3} a^{4} x}{4} \frac{d}{d x}\left(\frac{1}{\left(1-x^{2}\right)^{2}}-1\right) .
$$

Differentiating over $x$ and substituting $x$, we finally have an expression for 3-fold backward scattering:

$$
R_{3}(p, a)=p^{3} a^{4} \frac{a^{2}(1-p)^{2}}{\left(1-(a(1-p))^{2}\right)^{3}} .
$$

We can also determine the combinatory term for $l=2$ (5-fold backward scattering). First, we estimate range for $n_{1}$ : $0<n_{1}<N-1$. Then, for chosen $n_{1}, n_{2}$ can vary in the range $0<n_{2}<n_{1}$. For chosen $n_{1}$ and $n_{2}, n_{3}$ can vary in the range $0<n_{3}<N-n_{1}$. For chosen $n_{1}, n_{2}$, and $n_{3}$ we have a range for $n_{4} 0<n_{4}<n_{1}+n_{3}-n_{2}$. Finally, for chosen $n_{1}, n_{2}, n_{3}$, and $n_{4}$, we have one trajectory with $n_{5}=N-n_{1}-n_{3}$ and $n_{6}=N-n_{2}-n_{4}$. Thus, to calculate all combinations for given $N$ we have an expression

$$
W(2 N, 5)=\sum_{n_{1}=1}^{N-2} \sum_{n_{2}=1}^{n_{1}-1} \sum_{n_{3}=1}^{N-1-n_{1}} \sum_{n_{4}=1}^{n_{1}+n_{3}-1-n_{2}} 1 .
$$

After some easy transformations finally we have

$$
W(2 N, 5)=\frac{1}{12}(N-2)^{2}(N-1)(N-3) .
$$

Making the same substitutions and applying the same approach with differentiating of the series (see (A.2)-(A.3)) 4 times we can get the following expression for $R_{5}$ :

$$
\begin{aligned}
R_{5}= & \frac{p^{5} a^{6}}{12} \frac{x}{2} \frac{d}{d x} \\
& \times\left\{\frac{1}{2 x^{3}} \frac{d}{d x}\left\{\frac{x^{3}}{2} \frac{d}{d x}\left\{\frac{x}{2} \frac{d}{d x}\left\{\frac{1}{1-x^{2}}-1-x^{2}\right\}\right\}\right\}\right\} .
\end{aligned}
$$

After differentiating and substituting $x$ we finally get

$$
R_{5}=p^{5} a^{6} \frac{1+a^{2}(1-p)^{2}}{\left(1-(a(1-p))^{2}\right)^{5}} .
$$

Single, 3-fold and 5-fold backward scatterings are compared on Figure 7. It is easy to see that multiple backward scattering is negligible in the whole range of $g$ even in case of low absorption $(a=0.95)$. 


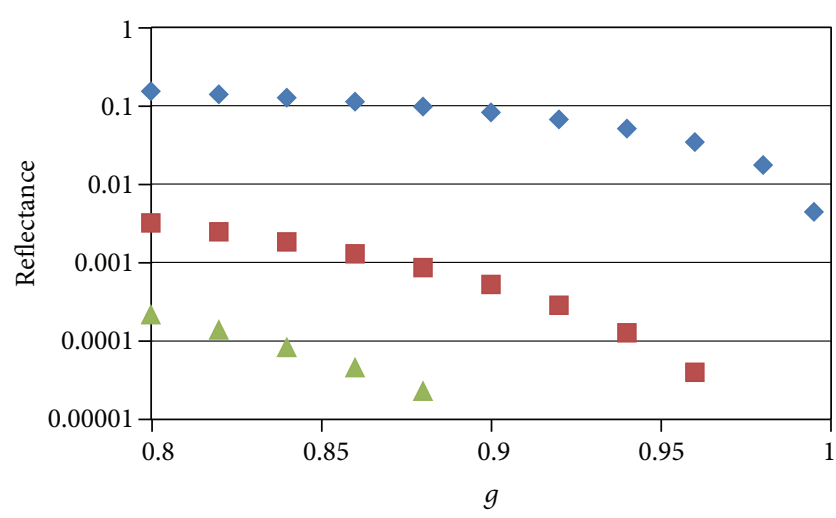

Figure 7: Dependence of single backward scattering (rhomboids), 3-fold (squares) and 5-fold (triangles) backward scattering as a function of $g(a=0.95)$.

\section{Conflict of Interests}

The authors declare that there is no conflict of interests regarding the publication of this paper.

\section{References}

[1] V. V. Tuchin, Tissue Optics: Light Scattering Methods and Instruments for Medical Diagnosis, vol. 166, SPIE Press, Bellingham, Wash, USA, 2nd edition, 2007.

[2] S. A. Prahl, M. Keijzer, S. L. Jacques, and A. J. Welch, "A Monte Carlo model for light propagation in tissue," in Dosimetry of Laser Radiation in Medicine and Biology, G. Muller and D. Sliney, Eds., vol. 5 of SPIE Series, pp. 102-111, 1989.

[3] S. A. Prahl, M. J. C. van Gemert, and A. J. Welch, "Determining the optical properties of turbid media by suing the addingdoubling method," Applied Optics, vol. 32, no. 4, pp. 559-568, 1993.

[4] A. Kienle, L. Lilge, M. S. Patterson, R. Hibst, R. Steiner, and B. C. Wilson, "Spatially resolved absolute diffuse reflectance measurements for noninvasive determination of the optical scattering and absorption coefficients of biological tissue," Applied Optics, vol. 35, no. 13, pp. 2304-2314, 1996.

[5] R. Reif, O. A'Amar, and I. J. Bigio, "Analytical model of light reflectance for extraction of the optical properties in small volumes of turbid media," Applied Optics, vol. 46, no. 29, pp. 73177328, 2007.

[6] L. T. Perelman, J. Wu, I. Itzkan, and M. S. Feld, "Photon migration in turbid media using path integrals," Physical Review Letters, vol. 72, no. 9, pp. 1341-1344, 1994.

[7] R. P. Feynman and A. R. Hibbs, Quantum Mechanics and Path Integrals, McGraw-Hill, New York, NY, USA, 1965.

[8] T. Harrison, J. C. Ranasinghesagara, H. Lu, K. Mathewson, A. Walsh, and R. J. Zemp, "Combined photoacoustic and ultrasound biomicroscopy," Optics Express, vol. 17, no. 24, pp. 2204122046, 2009.

[9] A. Kim and B. C. Wilson, "Measurement of ex vivo and in vivo tissue optical properties: methods and theories," in OpticalThermal Response of Laser-Irradiated Tissue, A. J. Welch, Ed., pp. 267-319, Springer, Dordrecht, The Netherlands, 2nd edition, 2011.
[10] S. C. Kanick, D. J. Robinson, H. J. Sterenborg, and A. Amelink, "Monte Carlo analysis of single fiber reflectance spectroscopy: photon path length and sampling depth," Physics in Medicine and Biology, vol. 54, no. 22, pp. 6991-7008, 2009.

[11] L. G. Henyey and J. L. Greenstein, "Diffuse radiation in the galaxy," Nature, vol. 147, no. 3733, pp. 613-626, 1941.

[12] G. Saiko and A. Douplik, "Real-time optical monitoring of capillary grid spatial pattern in epithelium by spatially resolved diffuse reflectance probe," Journal of Innovative Optical Health Sciences, vol. 5, no. 2, Article ID 1250005, 9 pages, 2012.

[13] P. Kubelka and F. Munk, "Ein beitragzur optik der farbanstriche," Zeitschrift für Technische Physik, vol. 12, pp. 593601, 1931.

[14] V. Džimbeg-Malčić, Ž. Barbarić-Mikočević, and K. Itrić, "Kubelka-Munk theory in describing optical properties of paper (I)," Technical Gazette, vol. 18, no. 1, pp. 117-124, 2011.

[15] L. Reynolds, C. Johnson, and A. Ishimaru, "Diffuse reflectance from a finite blood medium: applications to the modeling of fiber optic catheters," Applied Optics, vol. 15, no. 9, pp. 20592067, 1976.

[16] R. A. J. Groenhuis, H. A. Ferwerda, and J. J. T. Bosch, "Scattering and absorption of turbid materials determined from reflection measurements," Applied Optics, vol. 22, no. 16, pp. 2456-2462, 1983.

[17] T. J. Farrell, M. S. Patterson, and B. C. Wilson, "A diffusion theory model of spatially resolved, steady-state diffuse reflectance for the noninvasive determination of tissue optical properties in vivo," Medical Physics, vol. 19, no. 4, pp. 879-888, 1992.

[18] J. L. Saunderson, "Calculation of the color pigmented plastics," Journal of the Optical Society of America, vol. 32, no. 12, pp. 727$729,1942$. 

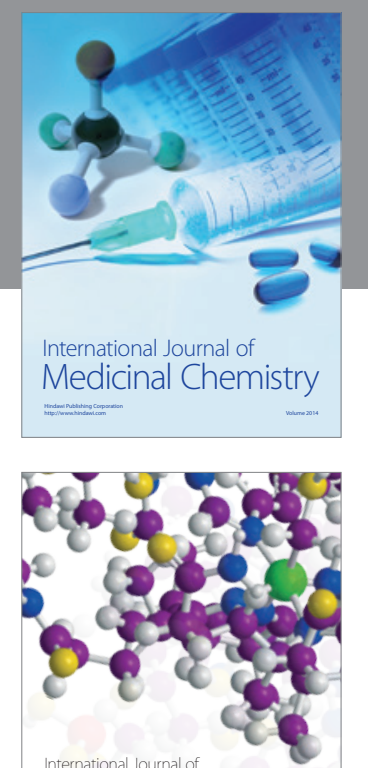

\section{Carbohydrate} Chemistry

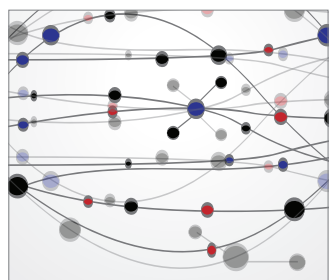

The Scientific World Journal
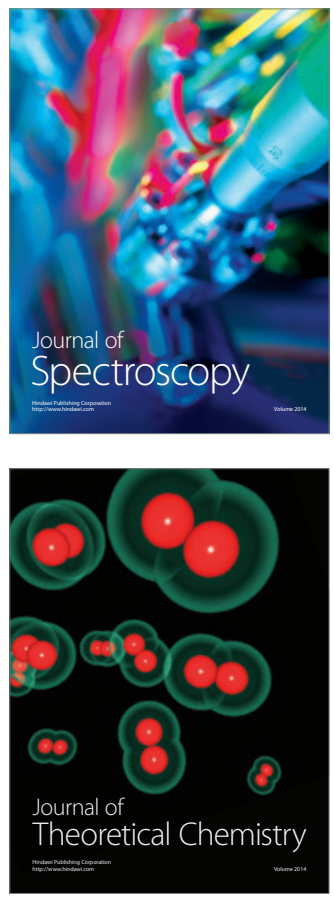
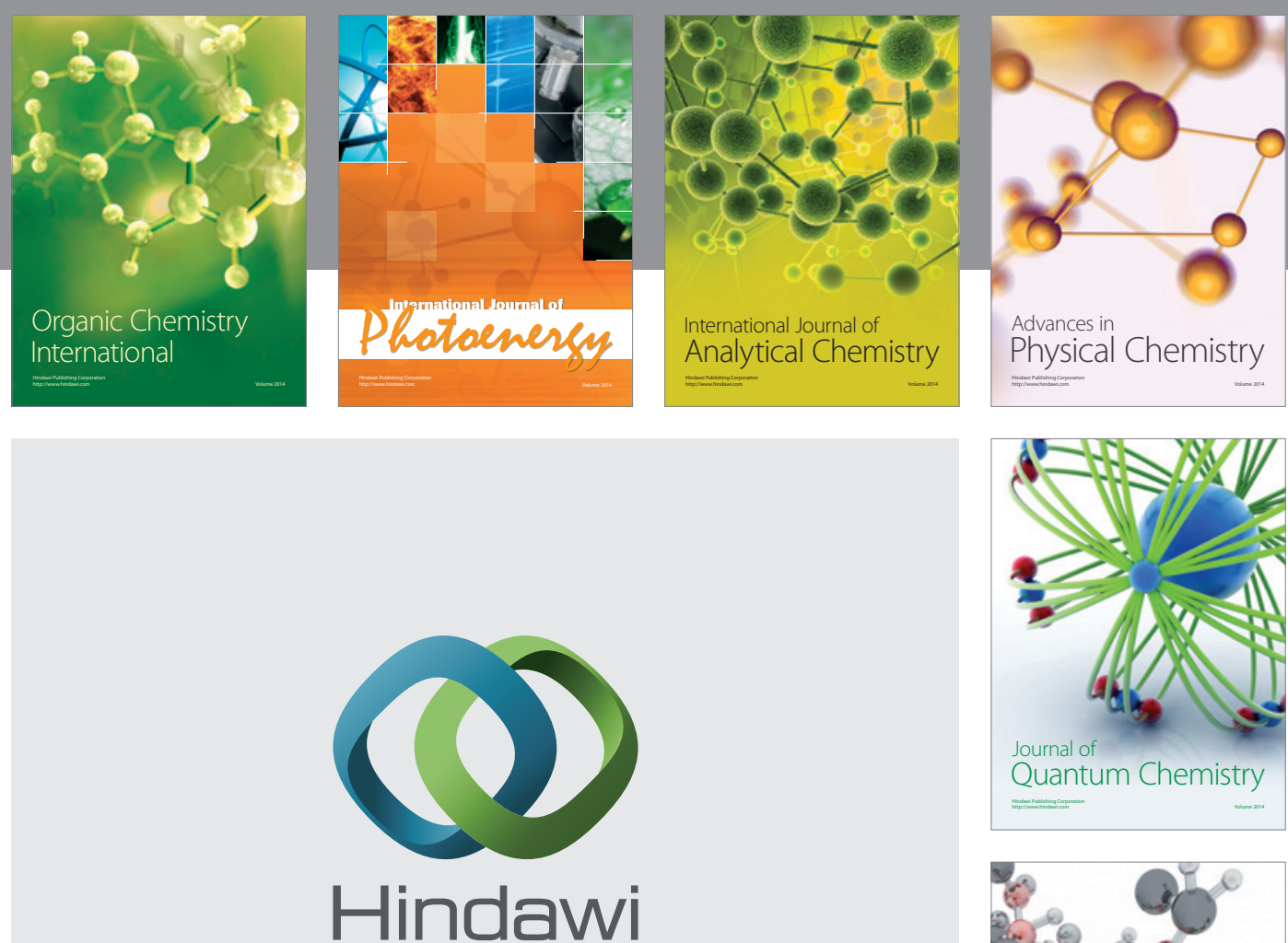

Submit your manuscripts at

http://www.hindawi.com

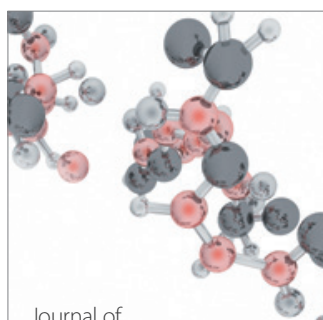

Analytical Methods

in Chemistry

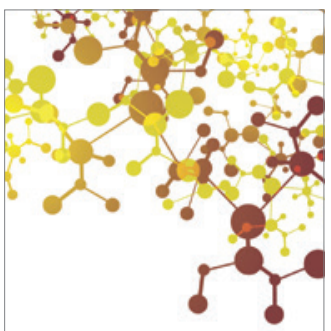

Journal of

Applied Chemistry

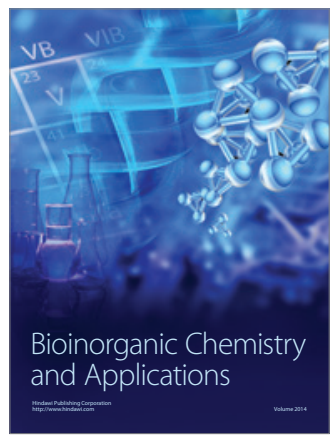

Inorganic Chemistry
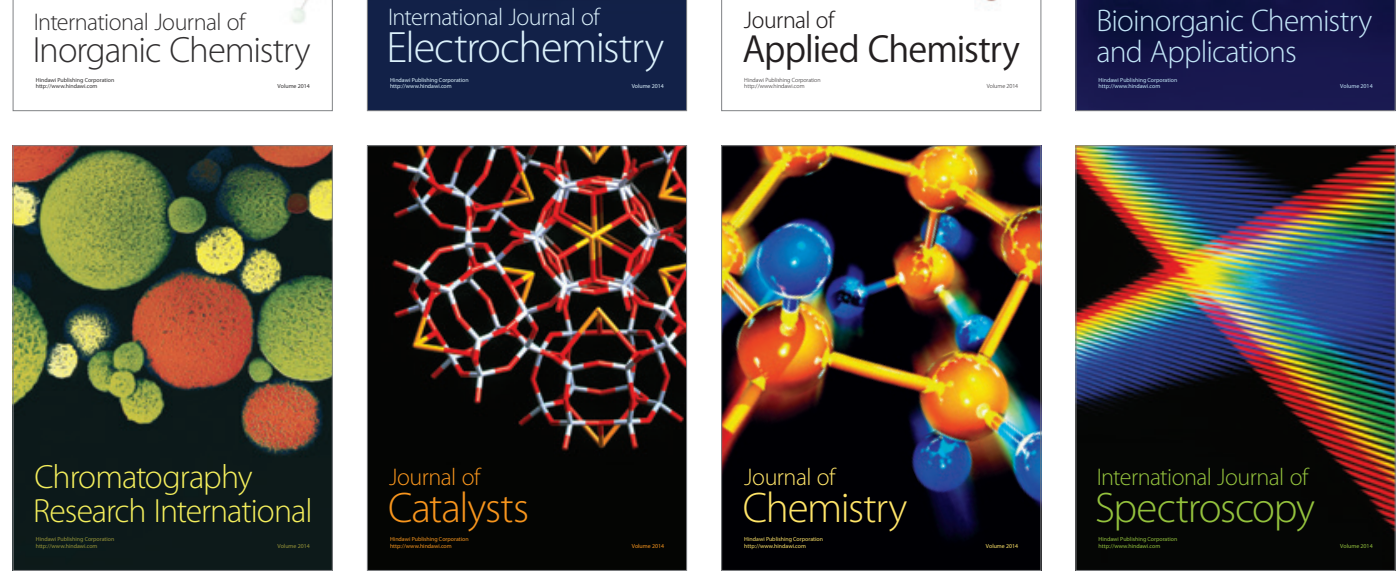